\title{
Intensive training in chronic upper limb hemiparesis does not increase spasticity or synergies
}

\author{
Annette Sterr, PhD; and Susanna Freivogel, PT
}

\begin{abstract}
The authors assessed whether intensive training increases spasticity and leads to the development of "pathologic movement patterns," a concern often raised by Bobath-trained therapists. The authors used a baseline-control repeated-measures test to study 29 patients with chronic upper limb hemiparesis who received daily shaping training. Their results suggest that training has no adverse effects on muscle tone and movement quality.
\end{abstract}

NEUROLOGY 2004;63:2176-2177

In most countries, physical and occupational therapy is the main treatment used for the rehabilitation of motor deficits after brain damage. The Bobath concept, known as neurodevelopmental treatment in the United States, ${ }^{1}$ is by far the most prominent school, ${ }^{1,2}$ with $88 \%$ of neurologic physiotherapists in the United Kingdom using the Bobath approach. ${ }^{3}$ However, insights into the mechanisms of brain plasticity, recovery, and learning have led to the development of new and (at least for a subgroup of conditions) potentially more effective training concepts that rely largely on the principles of repetition and massed practice. ${ }^{4}$ Such training-based interventions have been reported to achieve good clinical results (e.g., see Taub et al., ${ }^{4}$ Duncan et al., ${ }^{5}$ Sterr and Freivogel, ${ }^{6}$ and Woldag and Hummelsheim ${ }^{7}$.

Conceptually, training-based interventions conflict with the dogmas that form the Bobath approach. More specifically, the latter technique is centered on the assumption that enhanced muscle tone and consequential "pathologic movement patterns" or synergies are the major cause of the observed motor deficits in hemiparetic patients. Therefore, reduction or normalization of muscle tone represents the central aim of any therapeutic action and is to be achieved before more normal movement patterns can be introduced by means of facilitation. It is further believed that practicing movements under enhanced tone conditions will increase spasticity and effectuate the permanency of "pathologic movement patterns." ${ }^{2}$ From this conceptual framework arises the assumption that practice under increased tone conditions is contrainductive and thus should be avoided. Conversely, training-based interventions do not specifically address muscle tone issues, and, within limits, affected arm movements are practiced in a repetitive fashion even if muscle tone is enhanced.
Therefore, the majority of the neurologic physiotherapy workforce may be apprehensive of trainingbased interventions. In light of these concerns, we used an evidence-based approach to assess the effect of intensive training on muscle tone and movement quality.

Methods. A convenience sample of 29 patients (20 \pm 4 years; 12 women) with chronic hemiparesis after brain damage (15 traumatic brain injury, 7 cardiovascular accidents, 6 infantile cerebral paresis, and 1 subcortical brain tumor) participated in the study. All participants were in a chronic state (mean chronicity, 3.8 years; minimum, 12 months; maximum, 22.5 years). The main inclusion criteria were 1) minimum $10^{\circ}$ extension (against gravity) of wrist and one finger; and 2) Ashworth score ${ }^{8}>1$ in at least two joints. The research was conducted in an in-patient neurorehabilitation clinic and was approved by the local ethics committee.

The intervention comprised a repetitive training regimen ${ }^{6}$ that used the shaping principle of constraint-induced therapy. ${ }^{4} \mathrm{~Pa}-$ tients were trained individually to perform increasingly complex affected arm movements. Verbal feedback on task performance and positive reinforcement was given for the slightest improvement. If muscle tone increased so that it led to a substantial performance decrease, patients were given a short rest to stretch the hypertone muscles before practice resumed. Typically, 4 to 10 shaping tasks were used per session, and each task was trained for 8 to 15 minutes.

The study design comprised a repeated-measures design. All 29 participants were tested before (pre) and after (post) the intervention. Baseline values also were obtained for a subgroup of 12 participants ( 3 weeks before treatment). Teams of two blind control physiotherapists performed the assessment and scoring.

Flexion and extension spasticities were measured with the Ashworth scale ${ }^{8}$ for 1) finger, 2) wrist, 3) elbow, and 4) shoulder. The quality of movement (QOM) was obtained with a questionnaire-based instrument measuring "real-world" behavior (motor activity log $[\text { MAL }]^{9}$ ) and a laboratory-based assessment (Wolf Motor Function Test [WMFT] ${ }^{10}$ ). Furthermore, the amountof-use score of the MAL was used as an outcome parameter for functional improvement.

Ashworth scores were subjected to two-factorial analysis of variance (ANOVA), comprising the factors treatment (pre/post for $\mathrm{n}=29$; baseline/pre and pre/post for $\mathrm{n}=12$ ) and joint (shoulder, elbow, wrist, and finger). This analysis revealed a treatment effect $(\mathrm{F}[1,28]=4.6, p=0.01$; illustrated in figure 1$)$, indicating that

From the Department of Psychology (Dr. Sterr), University of Surrey, Guildford, UK; and Rehabilitation Clinic Hegau Jugendwerk (S. Freivogel), Gailingen, Germany.

Supported by the Medical Research Council, UK.

Received April 22, 2004. Accepted in final form July 22, 2004.

Address correspondence and reprint requests to Dr. Annette Sterr, Department of Psychology, University of Surrey, Guildford, GU2 7XH, UK; e-mail: a.sterr@surrey.ac.uk

2176 Copyright @ 2004 by AAN Enterprises, Inc. 


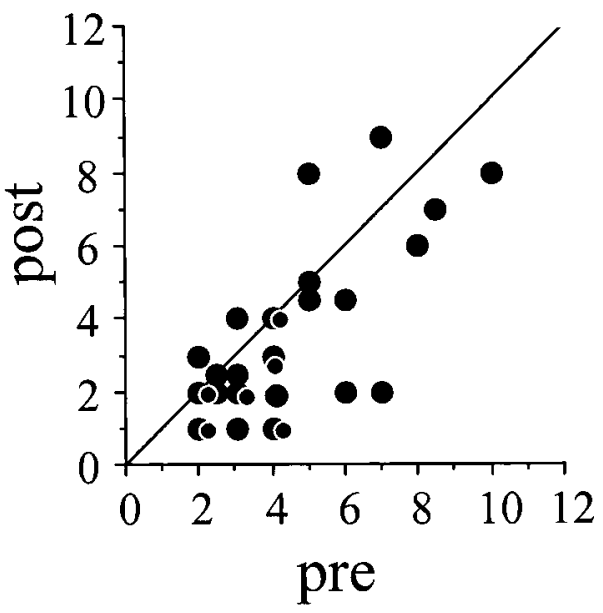

Figure 1. Bivariate scatterplot for summated Ashworth scores (shoulder + elbow + wrist + finger). Dots represent individual scores. Subjects who fall in the light gray area have lower scores at the post-assessment. Subjects who fall in the darker area showed increased spasticity at the posttesting. This is true for 4 of 29 participants (= 12\%; two stroke, one traumatic brain injury, and one cerebral palsy). Despite an overall increased Ashworth score in the patients, quality of movement scores in motor activity log and Wolf Motor Function Test were higher after the training in each of the four individuals.

spasticity scores decreased with training. Additionally, a joint effect was found $(\mathrm{F}[1,28]=5.6 ; p<0.05)$, showing that spasticity was most pronounced in the elbow joint. The subgroup analysis further indicated that baseline and pre-measures were not significantly different $(\mathrm{F}[1,11]=0.1 ; p=0.74)$, whereas the pre- to post-comparison revealed a trend for decreased spasticity $(\mathrm{F}[3,33]=$ $2.4 ; p=0.09$ ), especially in wrist and finger joints.

QOM scores were subjected to one-way ANOVA, which revealed treatment effects for the MAL $(\mathrm{F}[1,28]=40.7 ; p<0.01)$ and the WMFT $(\mathrm{F}[1,28]=37.7 ; p<0.01 ;$ both illustrated in figure 2). Again, baseline to pre-comparisons were insignificant (MAL: $\mathrm{F}[1,11]=0.2, p=0.68$; WMFT: $\mathrm{F}[1,11]=1.2, p=0.29$ ).

Everyday use of the affected arm also increased $(\mathrm{F}[1,28]=$ $37.8 ; p<0.01$; pre, $2.0 \pm 1.2$ points; post, $3.3 \pm 1.1$ points), which suggested that movement abilities improved with the training.

Results and discussion. Our results allay the concern that intensive affected arm training may increase muscle tone. Our data suggest that the shap-

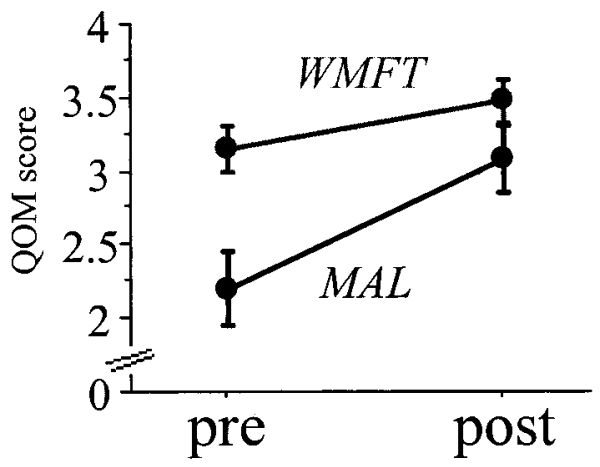

Figure 2. Quality of movement scores derived from the motor activity log questionnaire and the Wolf Motor Function Test before and after treatment. $Q O M=$ quality of movement. ing training might help reduce spasticity in chronic patients with lower-to-moderate tone enhancement. Furthermore, the increase of movement quality in both, the subjective MAL measure and the expert rating in the WMFT, provides empirical evidence against the dogma of "pathologic movement patterns." Thus, as long as the desired behavioral goal could be achieved, the training regimen encouraged patients to practice goal-directed affected arm movements despite the presence of enhanced tone and synergistic movement patterns. However, this procedure did not enhance synergies, as the theory of pathologic movement patterns would predict, but led to a gradual normalization of muscle tone and movement patterns.

Our data suggest that intensive training in patients with increased muscle tone improves function without exacerbating spasticity and synergies. This evidence is not compatible with the underlying assumptions of the Bobath approach. Despite enhanced tone and without the therapist's "facilitation of more normal movement patterns," patients can regain functional abilities if they are given the chance to practice in a repetitive and demanding fashion. This is also true in a case in which a transient increase of spasticity occurs during the training sessions. Interestingly, four patients who showed higher Ashworth scores in the post-treatment assessment still improved in the clinical outcome measures. Therefore, the common practice of advising patients to refrain from performing functional tasks as long as muscle tone has not normalized ${ }^{2}$ should be reconsidered. Electrophysiologic measurement of adventitious and synergistic muscle fascilitation will be necessary to prove the absence/diminuation of inadvertent exaggerations of inappropriate musculature after training.

\section{Acknowledgment}

The authors thank Amy Saunders for editorial assistance.

\section{References}

1. Lennon S, Ashburn A. The Bobath concept in stroke rehabilitation: a focus group study of the experienced physiotherapists' perspective. Disabil Rehabil 2000;22:665-674.

2. Lennon S, Baxter D, Ashburn A. Physiotherapy based on the Bobath concept in stroke rehabilitation: a survey within the UK. Disabil Rehabil 2001;23:254-262.

3. Davidson I, Waters K. Physiotherapists working with stroke patients. Physiotherapy 2000;86:69-80.

4. Taub E, Uswatte G, Elbert T. New treatments in neurorehabilitation founded on basic research. Nat Rev Neurosci 2002;3:228-236.

5. Duncan PW. Synthesis of intervention trials to improve motor recovery following stroke. Topic Stroke Rehabil 1997;3:1-20.

6. Sterr A, Freivogel S. A practical version of CI therapy for the clinical environment. Neurology 2003;61:842-844.

7. Woldag H, Hummelsheim H. Evidence-based physiotherapeutic concepts for improving arm and hand function in stroke: a review. J Neurol 2002;249:518-528.

8. Ashworth B. Preliminary trial of carisoprodol in multiple sclerosis. Practitioner 1964;192:540-542.

9. Taub E. Overcoming learned nonuse. A new approach to treatment in physical medicine. In Carlson JG, Seifert AR, Birbaumer N, editors. Clinical applied psychophysiology. New York: Plenum Press, 1994:185220

10. Morris DM, Uswatte G, Crago JE, Cook EW III, Taub E. The reliability of the Wolf Motor Function Test for assessing upper extremity function after stroke. Arch Phys Med Rehabil 2001;82:750-755. 